\title{
PAPER
}

\section{Quantitative analysis of the velocity related pathophysiology of spasticity and rigidity in the elbow flexors}

\author{
H-M Lee, Y-Z Huang, J-J J Chen, I-S Hwang
}

See end of article for authors' affiliations .....................

Correspondence to: Professor Jia-Jin J Chen, Institute of Biomedical Engineering, National Cheng Kung University, Tainan 701, Taiwan; ROC; jason@jason.bme.ncku.edu.tw

Received

29 October 2001

In revised form

9 January 2002

Accepted

18 January 2002
Objective: To quantify velocity dependent and position related properties of increased muscle tone measured during a constant velocity stretch.

Methods: Elbow flexors were vertically stretched under four different velocities $140,80,120$, and $160^{\circ} / \mathrm{s}$ ) through a $75^{\circ}$ range of motion in 12 patients with hemiparesis, 16 with parkinsonism, and 12 normal controls. From reactive torque measurement, a linear second order model was adopted to dissociate velocity dependent viscous and velocity independent elastic components. The averaged speed dependent reflex torque (ASRT) - defined as the deviation of measured torque from baseline torquewas used to quantify the viscous component of hypertonia. Velocity sensitivity of ASRT (VASRT) and segmented ASRT (SASRT), derived from the slope of the regression line among ASRT velocity plots and from segmentations of reactive torque, respectively, were used to differentiate the increased muscle tone of spasticity and rigidity.

Results: ASRT and VASRT were significantly higher in both spasticity and rigidity than in normal controls. SASRT analysis showed three different position related patterns among spasticity, rigidity, and normal groups: spasticity showed progressively increasing muscle tension relative to position; rigidity showed increased (relative to the norm) but constant muscle tone over the entire stretch range; the normal control group showed a consistently low reactive torque over the entire range.

Conclusions: Velocity dependence analysis indicates that rigidity and spasticity have approximately equal velocity dependent properties. For differentiating these two types of hypertonia, position dependent properties my be employed.
S haring the clinical feature of increased muscle tone, spasticity and rigidity are major motor and movement disorders in subjects with lesions of the upper motor neurones. In clinics, differentiating spasticity and rigidity has practical importance in the diagnosis and management of altered muscle tone. Various types of neurophysiological and biomechanical approach have been developed for quantitative investigation of the neural control of muscle tone. One common approach is to observe the sensitivity of muscle resistance in response to stretch velocity or displacement, which is then used to infer the underlying pathophysiological mechanism. ${ }^{1-3}$ As stretch movement is a function of stretch velocity, the velocity factor has played an indispensable role in characterising muscle response to stretch and in differentiating spasticity from rigidity.

Arising from hyperexcitability of the stretch reflex, spasticity has been defined as a velocity dependent increase in muscle tone. ${ }^{4}$ Usually, two major velocity dependent characteristics can be observed by stretching a spastic limb at different velocities. First, sufficiently fast stretch velocity is essential to induce spasticity. The velocity of passive displacement must exceed a certain value, otherwise neither an increase in muscle tone nor a reflexive electromyogram (EMG) can be observed. Researchers have reported that even a stretch velocity of $35^{\circ} / \mathrm{s}$ induces a considerable stretch reflex, with marked EMG activity, in a spastic elbow flexor. ${ }^{5}$ Second, the reactive muscle response increases in proportion to stretch velocity. EMG and torque measurements during ramp and hold stretches have shown this velocity dependent feature in various spastic joints. Reflexive EMG activity in the passive spastic elbow flexor has been shown to have a linear dependence on the ramp velocity over the range of 15 to $300^{\circ} / \mathrm{s}^{56}$ Torque measurement of the spastic knee joint — either maximum torque or sum of torque-was found to increase with the increase in stretch velocity. ${ }^{7}$ In addition, average torque during passive dorsiflexion was proportional to the angular velocity and correlated well with clinical assessment of hypertonia in the spastic ankle joint. ${ }^{8}$

Compared with spasticity, the pathophysiological mechanisms of parkinsonian rigidity are less well understood. Parkinsonian rigidity is considered to be independent of hyperactive segmental stretch reflexes, so that tendon jerks and $\mathrm{H}$ reflex behaviour seem almost normal in subjects with parkinsonism. ${ }^{9-11}$ From clinical observations, it is generally known that resistance in a rigid limb persists over the entire stretch range and is present even during very slow velocity stretch. ${ }^{12}$ Previous studies have reported that the contribution of elastic stiffness in parkinsonian rigidity is greater than in normal muscle. ${ }^{13}{ }^{14}$ These findings suggest that the increased stiffness might be attributed to mechanical changes in passive properties of muscle. As a result, the increase in stiffness has generally been used as a quantifying index in assessing the severity of rigidity ${ }^{14-16}$ These observations imply that rigidity is relatively velocity independent, so the contribution of velocity factor in the genesis of parkinsonian rigidity has not been much studied.

With a goal of characterising spasticity and rigidity, we were interested in comparing the velocity dependent components of muscle tone using constant stretch at various velocities. From a methodological viewpoint, responsive torque measurement during constant velocity stretch is a more direct method of recording muscle tone than EMG recordings or other neurophysiological measurements. ${ }^{17}$ However, erroneous recordings during torque measurement caused by poor limb relaxation, inappropriate positioning, and the various inertial components of the system can limit the applicability of the stretch torque 
method. In our previous study, ${ }^{18}$ we developed an on-line muscle tone measurement system which systematically alleviated potential errors of stretch torque measurement and was successfully applied to evaluating the time course changes of spasticity in stroke patients. ${ }^{18}$ We based the present study on our previous motor driven torque measurement apparatus, aiming to characterise the velocity related properties of hemiparetic spasticity, parkinsonian rigidity, and normal muscle tone. We also used the change in reactive torque with displacement to observe the position related properties of hypertonic conditions during four constant velocity stretches. We discuss the underlying pathophysiology differentiating spasticity from rigidity as determined by measurements of reactive torque under constant velocity stretch.

\section{METHODS}

\section{Subjects and clinical evaluation}

We recruited three groups of subjects for the study, representing spasticity, rigidity, and normal controls. Twelve subjects had spastic hemiparesis, age range 31 to 77 years (mean (SD), 62.2 (6.5) years), with time from onset varying from 8 weeks to 20 years. Sixteen had rigid parkinsonism, age range 27 to 85 years (mean 60.6 (13.5)) and time of onset between 4 weeks and 6 years. The control group consisted of 12 age matched normal persons without a history of neurological disease.

All the subjects gave their informed consent before they participated in the study, which was approved by the local ethics committee on human experiments of National Cheng Kung University Hospital.

All subjects with neurological disease were diagnosed and evaluated by a qualified neurologist ( the first coauthor). Criteria for inclusion included having a stable neurological condition, sufficient cognitive ability to be cooperative, and a sufficient range of motion around the elbow joint, without contracture. Before the tests, the patients were asked not to take any drugs for at least 12 hours, to ensure steady hypertonia. A routine neurological evaluation, including assessment of the biceps tendon reflex and muscle tone, was undertaken before the test trials. For clinical assessment, the modified Ashworth scale (0-5) was used for spastic subjects, while item 22 of the unified Parkinson's disease rating scale (0-4) was used to evaluate rigidity. The affected side of the hemiparetic patients or the more severely affected side of the parkinsonian patients was chosen for the stretch tests. Owing to the potential abnormality of muscle tone in the unaffected side of hemiparetic subjects, the dominant limb of the normal subjects was selected as the reference, as suggested before. ${ }^{19} 20$

\section{Procedures and instrumentation}

During stretch experiments, subjects lay supine on a bed in a quiet, temperature controlled room so that they should be as comfortable and relaxed as possible. Tests were taken with slight abduction of the upper arm (about $\left.5-10^{\circ}\right)$, and with the forearm strapped to the apparatus, which was operated by a motor (DM1150A50, Yokogawa Electric Corporation, Tokyo, Japan) and was connected to a torque sensor (SRM3-6-1000, Advanced Mechanical Technology, Watertown, USA). The stretching motion was in the vertical plane. The torque sensor was firmly mounted on the rotational centre of the motor, and hence the reactive torque from the elbow joint was directly recorded during stretch. The details of the motor driven torque measurement system are given in a previous paper. ${ }^{18}$

For the vertical ramp and hold stretch we chose a relatively large $75^{\circ}$ displacement, from $105^{\circ}$ to $30^{\circ}$ elbow flexion $\left(0^{\circ}\right.$ flexion is assumed to be the fully extended position). This stretch range covers most of the normal movement range, but did not exceed the maximum range of movement for any subject in the study. Muscle activity in the biceps and triceps was recorded from $\mathrm{Ag}-\mathrm{AgCl}$ disc surface electrodes. These data, along with the torque signal data, were sent to a personal

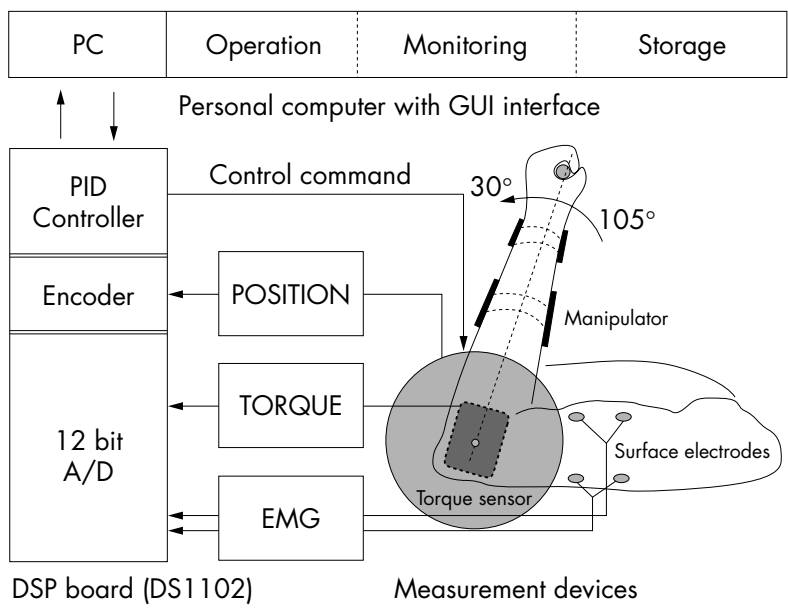

Figure 1 A block diagram of the motor driven muscle tone measurement system. The measurement devices include a motor with position encoder, torque sensor, and surface EMG electrodes for monitoring muscle activity. Control of constant stretch relies on the controller in the digitolvisual processor (DSP) board, which also transmits the stretch responses, including torque and EMG signal, through an analogue to digital converter to a personal computer. Graphic user interface in of the personal computer (PC) is capable of initiating motor rotation as well as monitoring and storing stretch responses during testing graphic user interface (GUI).

computer through an analogue to digital converter (12 bit resolution, $2 \mathrm{kHz}$ sampling rate). The experimental set-up is shown schematically in fig 1 .

Four stretch velocities-of 40,80, 120, and $160^{\circ} / \mathrm{s}$ in random sequence-were applied once each per test session. For normal subjects, all stretch velocities were below the critical velocity $\left(175^{\circ} / \mathrm{s}\right)$ so as not to induce the stretch reflex ${ }^{5}$ or alter the level of muscle resistance in relaxed elbow flexors. In all, five repetitive test sessions-with each velocity sequence randomised afresh per test session-were undertaken over approximately one hour in the selected limb to reduce intrasubject variability.

\section{Quantification analysis}

Torque measurement of the velocity related properties of muscle tone requires a quantitative index for extracting and representing the velocity dependent viscous component of muscle tone. Several factors-including the inertia resulting from the acceleration/deceleration of the stretch motor, gravitational effects of limb and attachment, and reactive muscle resistance from the limb-could contribute to the measured torque. To model the measured torque, a common approach is to derive the inertial (I), viscous (B), and elastic stiffness (K) components, and also the constant offset $(\mathrm{C})$ including gravitational torque and other influences from the second order biomechanical model, as described in equation $(1)^{21}$ :

$$
\mathrm{Tv}=\ddot{I} \ddot{\theta}+\mathrm{B} \dot{\theta}+\mathrm{K} \theta+C
$$

where $T_{v}$ is the measured torque at stretch velocity $v$ and $\theta$ is the angular displacement. However, to extract only the viscous component of muscle tone, one needs to identify the accurate inertial torque, elastic torque, and gravitational torque during each stretch. In our previous study, we proposed an empirical approach for circumventing the complexities involved in the exact modelling of stretched muscle and motor inertia. ${ }^{18}$

Figure $2 \mathrm{~A}$ shows the typical measured torque (solid line) of a spastic hemiparetic patient during $80^{\circ} / \mathrm{s}$ stretch velocity. By focusing only on the torque over the constant velocity range of the stretch (from Pl to P2), the sinusoidal-like inertia components outside of $\mathrm{P} 1$ and $\mathrm{P} 2$ are not taken into account,

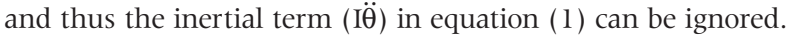




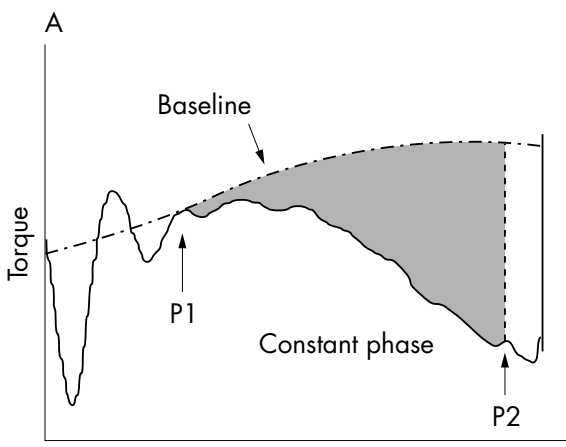

Displacement

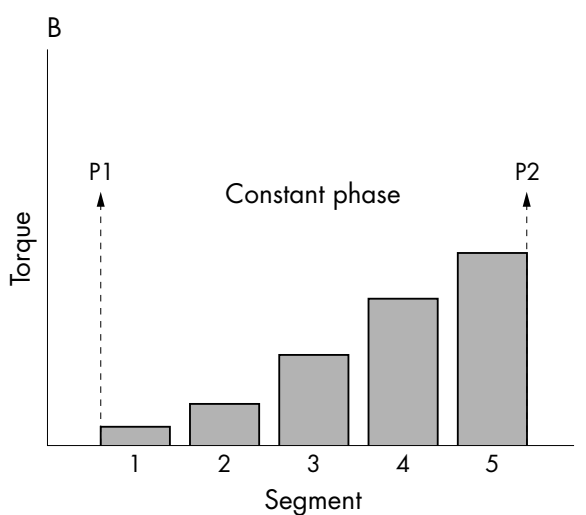

Figure 2 (A) An example of the reactive torque (continuous line) measured at $80 \%$ s plotted against the baseline torque (dot-dash line) obtained from a very slow stretch velocity of $5 \%$. The shaded area between baseline torque and measured torque during the constant velocity phase (P1-P2) represents the velocity dependent component of reactive muscle tone. (B) Segmentation of reactive torque during the constant period between $\mathrm{P} 1$ and $\mathrm{P} 2$ shows a position related pattern during constant velocity stretch.

To observe only the velocity dependent viscous component (Bं் in equation ( 1$)$ ), the elastic component $(\mathrm{K} \theta)$ as well as the gravitational effects on the stretched limb and the manipulator (C) should be avoided. At very slow velocity, the muscle resistance induced by the velocity factor is trivial, but the gravitational effect remains the same as for higher stretch velocities. Thus the torque measured (dashed line in fig $2 \mathrm{~A}$ ) during a very slow stretch velocity $-5^{\circ} / \mathrm{s}$ in this study-can be defined as baseline torque. Essentially, the baseline torque represents the elastic and gravitational parts of the measured torque during stretch. Hence, after subtracting the baseline torque from the high velocity torque, the shaded area during constant phase can be extracted as the velocity dependent component of reactive torque (as shown in fig $2 \mathrm{~A}$ ). In practice, by transforming the time unit to stretch angle, the fitted torque curve can be integrated with respect to the stretch angle. As the time between $\mathrm{P} 1$ and $\mathrm{P} 2$ varies with test subject and stretch velocity, the integration of the shaded area is then divided by the angular displacement. The normalised area (that is, the averaged amplitude of the shaded area)—defined as averaged speed dependent reflex torque (ASRT) - is used for quantifying the velocity dependent component of increased muscle tone.

To avoid undesired voluntary contraction during baseline stretch and to ensure the quality of the baseline torque data, three approaches were taken. First, an on-line EMG was monitored to ensure that the tested arm was relaxed before and during the test sessions. Second, measured torques were observed on-line to prevent misalignment or voluntary contraction during stretch. Third, the variation in repeated baseline torque measurements was analysed, as high reproducibility guarantees the validity of the baseline torque measurements. The reproducibility of baseline torque measurements for each subject was evaluated by the variation ratio (VR), ${ }^{22}$ ranging from 0 to 1 . Perfect reproducibility of several torque profiles results in zero VR. Panels A and B in fig 3 show two typical examples of high and low baseline torque reproducibility as estimated by VR. A high VR, as in fig 3B-indicating low reproducibility-is assumed to indicate an invalid stretch resulting from the muscle not fully relaxing during stretch.

Two additional indices for describing velocity dependence and position dependence of reactive torque (VASRT and SASRT) were derived as follows. The ASRT values measured at four stretch velocities were combined to observe the velocity dependence of the reactive torque. For each subject, the slope of the linear regression line for 20 ASRT values at four stretch velocities was designated as the velocity sensitivity of ASRT score (VASRT). As the ASRT is proportional to the viscous component (B) $)$, VASRT approximates the ASRT increase with respect to stretch velocity and becomes a variable related to viscosity. Reactive torque with respect to stretch position is also an important feature for quantifying muscle tone. However, ASRT is derived by integration over a constant velocity range, such that the detailed pattern related to a particular stretch position is averaged out. Thus to reveal position related patterns of ASRT, we divided the total shaded area displacement into five segments or subranges, as illustrated in fig 2B. The segmented ASRT (SASRT) represents the averaged amplitude of the reactive torque for the five displacement ranges. SASRT can be used to characterise the position related pattern of reactive torque during constant velocity stretching.

\section{Data analysis}

Intragroup and intergroup analyses of the three reactive torque parameters-ASRT, VASRT, and SASRT-were undertaken for hemiparetic, parkinsonian, and normal groups.
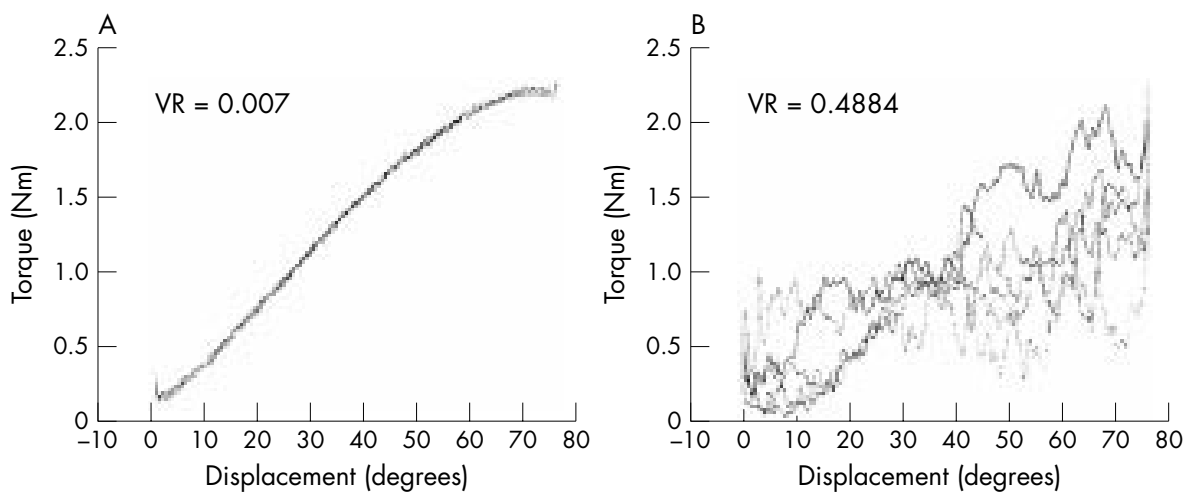

Figure 3 (A) Typical low variability of baseline torques with $V R=0.007$, indicating good repeatability without undesired human intervention. (B) A higher VR value results from an unrelaxed stretched limb or voluntary contraction during stretches. 
Table 1 Summary of clinical scales and tested limbs for hemiparetic and parkinsonian subjects

\begin{tabular}{|c|c|c|}
\hline Subject & Clinical scales/ number of subjects & Tested limb (right, left) \\
\hline $\begin{array}{l}\text { Hemiparesis }(n=12) \\
\text { Parkinsonism }(n=16)\end{array}$ & $\begin{array}{l}\operatorname{MAS}(1,2,3,4) /(3,4,4,1) \\
\operatorname{UPDRS}(1,2,3) /(5,8,3)\end{array}$ & $\begin{array}{l}(7,5) \\
(9,7)\end{array}$ \\
\hline
\end{tabular}

Twenty torque measurements, including five repetitive tests at four stretch velocities, were recorded for each subject. Cumulatively, a total of 800 ASRT values were collected. For ASRT analysis, a subject was exposed in turn to each condition (four stretch velocities), and hence the stretch velocity was a factor with repeated measures. A two way repeated measures analysis of variance (ANOVA) was performed for the 160 averaged ASRT values to test group differences and velocity effects. For each subject, VASRT was derived from 20 ASRT values from four stretch velocities. Forty VASRT values were then tested by simple one way ANOVA to examine VASRT differences among the three groups. Similarly, the position factor in the SASRT analysis was also a repeated measures factor. To test group dif-
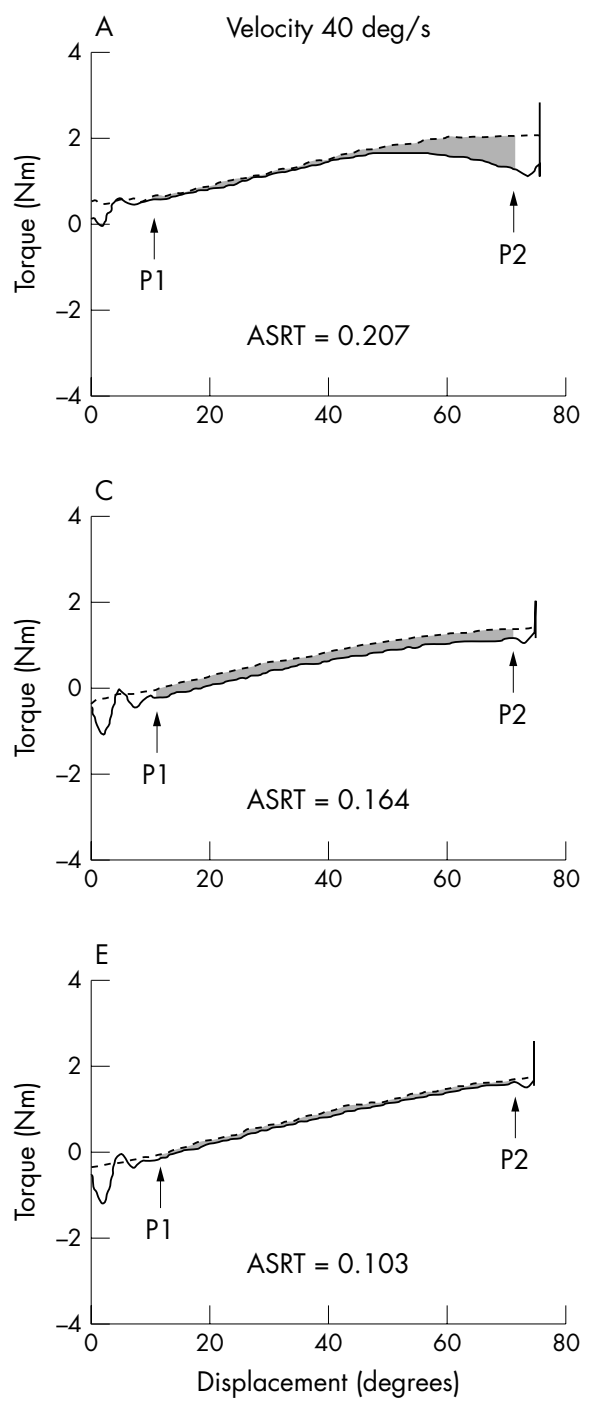

Figure 4 Representative examples of velocity dependent components of reactive torque (solid line) deviated from baseline torque (dashed line) for hemiparetic $(A, B)$, parkinsonian $(C, D)$, and normal subjects $(E, F)$, recorded at low $\left(40^{\circ} / \mathrm{s}\right)$ and high $\left(120^{\circ} / \mathrm{s}\right)$ stretch velocities. A significant increase in averaged speed dependent reflex torque (ASRT) (normalised shaded area) was noted in both hemiparetic and parkinsonian subjects.

ferences and position effects for SASRT, data from 40 SASRT measurements were examined by a two way ANOVA test with repeated measures in each velocity. More detailed comparison or a posteriori testing was performed if further analysis was necessary. Only probability $(p)$ values of $<0.01$ were regarded as significant in these tests. Values are given as mean (SD).

\section{RESULTS}

The modified Ashworth scale for the 12 hemiparetic subjects and the unified Parkinson's disease rating scale for the 16 subjects with parkinsonism, and information on which limbs were tested, are given in table 1 . No significant bias was noted in the clinical scales and in the sides tested. To confirm the
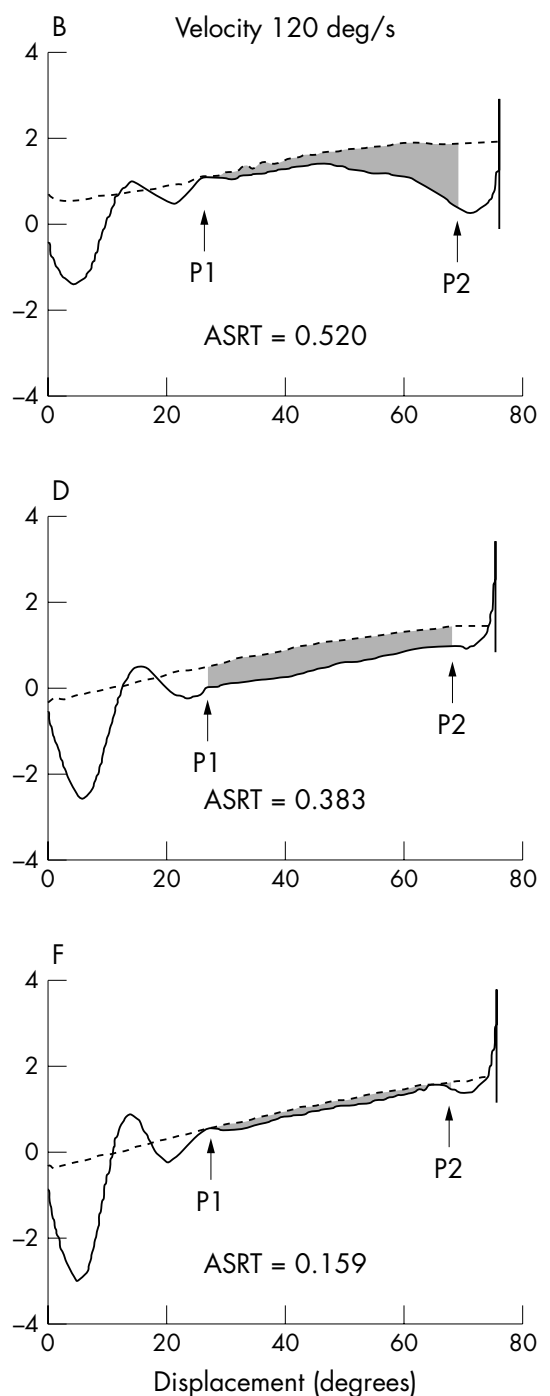
Table 2 Two way analysis of variance for ASRT analysis

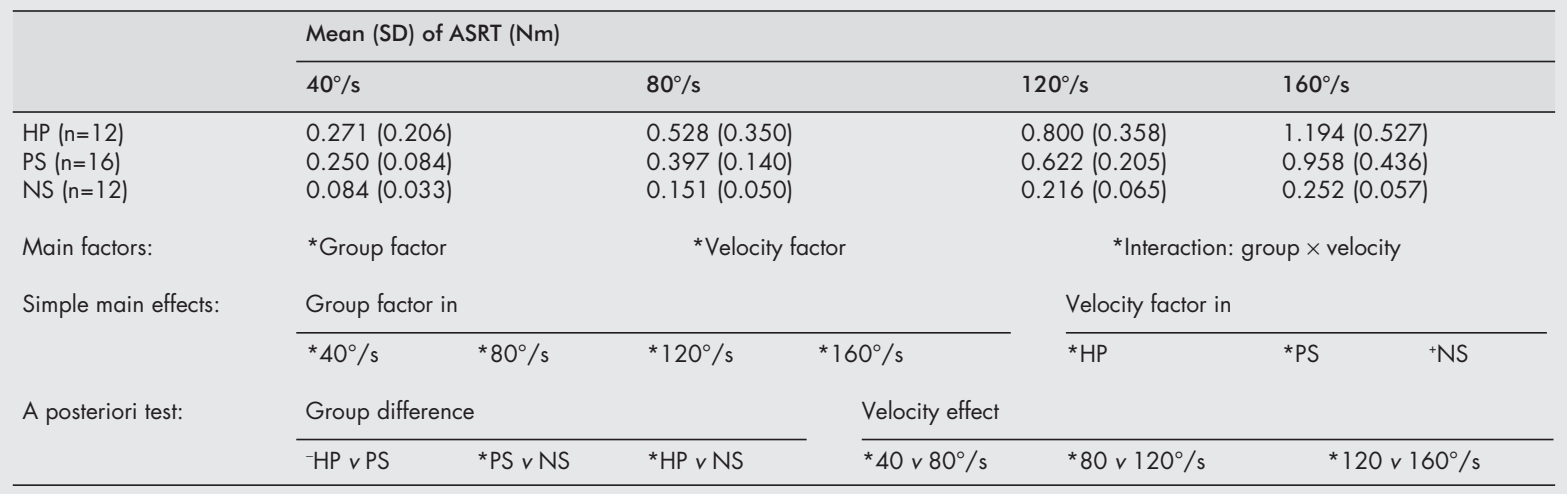

All tests marked with asterisk show significant difference $(p<0.01)$ in statistical comparisons, except the velocity factor in normal group (marked " + ") and the a posteriori test between parkinsonian and hemiparetic subjects (marked "-").

ASRT, averaged speed dependent reflex torque; HP, hemiparesis; NS, normal subject; PS, parkinsonism.

measurement validity of the baseline torques, variation in the five baseline torques was evaluated off-line using the VR index. Our VR analysis indicated a very high reproducibility in normal and hemiparetic patients (normal, 0.0059 (0.0051); hemiparesis, $0.0074(0.0067)$ ). A slightly higher but still very low variability was observed in parkinsonian patients $(0.0470$ $(0.0051))$.

\section{Quantification of viscous component of muscle tone using ASRT}

Figure 4 shows a comparison of reactive torque measured at various stretch velocities in typical hemiparetic, parkinsonian, and normal subjects. For clarity, only the reactive torque of the lowest and the highest stretch velocities-that is, $40 \% \mathrm{~s}$ and $120^{\circ} / \mathrm{s}$ - are shown in this comparison. Panels A-D show obvious deviation of reactive torque (solid line) from baseline torque (dashed line) in the hemiparetic and parkinsonian subjects, with ASRT values increasing as the deviation increases. Moreover, the ASRTs at the higher velocity $\left(120^{\circ} / \mathrm{s}\right)$ were obviously higher than those at the lower velocity $(40 \%)$. In contrast, consistently low ASRT values were found in the normal subjects for both stretch velocities, as shown in panels $\mathrm{E}$ and $\mathrm{F}$.

ASRT values for all measurements were then pooled to determine the relations of ASRT to group differences and stretch velocities by repeated measures ANOVA. Table 2 summarises the ANOVA test results, including the main factors and detailed comparisons. On the whole our results show that both group and stretch velocity were important factors in the ASRT measurements $(\mathrm{p}<0.01)$.

To gain a better understanding of the details of the ASRT distribution among the three groups and four velocities, further analyses was performed as follows. As the interaction between the two main factors was significant, the simple main effects were first evaluated to weigh the effects of the main factor at each level. Analysis of the group factors for the four stretch velocities showed that ASRT group differences were significant for all velocities $(p<0.01)$. However, a posteriori comparison using Tukey's honestly significant difference (HSD) method showed that group differences were present only in the parkinsonism/normal and hemiparesis/normal pairs (PS $v$ NS and HP $v$ NS in table 2). These results indicate that ASRT was able to distinguish clearly between normal and pathological muscle tone, but failed to reveal the difference between spasticity and rigidity. On the other hand, analysis of the velocity factor for the three groups-as shown in the lower right part of table 2-indicates that ASRT differences resulting from velocity effects were significant for the hemiparetic and parkinsonian groups $(p<0.01)$, but not for the normal subjects $(p>0.05)$. To observe the stretch velocity effect on ASRT values, the detailed stretch velocity differences were also evaluated with the a posteriori Tukey HSD comparison. Significant differences $(\mathrm{p}<0.01)$ were found in pairs of $40-80 \%$ s, $80-120 \%$ s, and $120-160 \%$ s. These observations indicate that increased reactive torque accompanied by increased stretch velocity were found in both hemiparetic spasticity and parkinsonian rigidity, but not in normal muscle tone.

\section{Velocity dependence of ASRT}

To analyse the velocity dependent properties of increased muscle tone, the variable VASRT-reflecting ASRT velocity dependence-was compared among the three groups. Figure 5 shows the ASRT velocity dependence for all the subjects, with the slope of the regression line representing the averaged VASRT for each group. Compared with the normal controls, higher slopes were noted in the hemiparetic and parkinsonian groups. The averaged VASRT values for the three subject groups are given in table 3. One way ANOVA testing showed that group differences were significant for VASRT analysis $(p<0.01)$. Further a posteriori comparison showed significant differences between hemiparetic/normal and

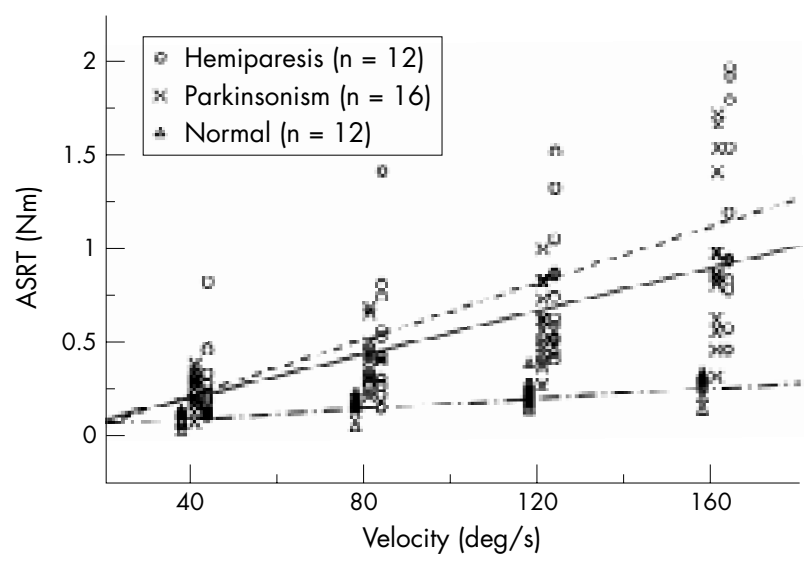

Figure 5 Comparison of pooled averaged speed dependent reflex torque values (ASRT) $v$ velocity in the hemiparetic subjects (circles), the parkinsonian subjects (crosses), and the normal controls (triangles). Each point represents an averaged ASRT value during a stretch velocity measurement. Slopes of regression lines (dashes for hemiparesis, continuous line for parkinsonism, and dot-dash for normal subjects) indicate the averaged velocity sensitivity of ASRT for each group. 
Table 3 One way analysis of variance for VASRT analysis

\begin{tabular}{|c|c|c|c|c|}
\hline & \multicolumn{3}{|c|}{ Mean (SD) VASRT $\left(\mathrm{Nm} /{ }^{\circ} / \mathrm{s}\right)$} & \multirow{2}{*}{$\begin{array}{l}\text { Group } \\
\text { difference }\end{array}$} \\
\hline & $\mathrm{HP}(n=12)$ & PS $(n=16)$ & NS $(n=12)$ & \\
\hline A posteriori test: & $\begin{array}{l}0.0076(0.0032) \\
H P \vee P S \\
p>0.05\end{array}$ & $\begin{array}{l}0.0057(0.0033) \\
H P \vee N S \\
p<0.01\end{array}$ & $\begin{array}{l}0.0014(0.0005) \\
P S \vee N S \\
p<0.01\end{array}$ & $p<0.01$ \\
\hline $\begin{array}{l}\text { Among the tests, } \\
\text { difference. } \\
\text { HP, hemiparesis; } \\
\text { dependent reflex }\end{array}$ & $\begin{array}{l}\text { S, normal subject; } P \\
\text { rque. }\end{array}$ & $\begin{array}{l}\text { he hemiparesis/p } \\
\text { rkinsonism; VASR }\end{array}$ & $\begin{array}{l}\text { sonism pairs showe } \\
\text { ocity sensitivity of }\end{array}$ & $\begin{array}{l}\text { gnificant } \\
\text { d speed }\end{array}$ \\
\hline
\end{tabular}

parkinsonian/normal pairs, but no differences between hemiparetic and parkinsonian groups $(p>0.05)$. The results indicate that muscle tone velocity dependence increased approximately equally in the hemiparetic and parkinsonian groups.

\section{Position dependence of SASRT}

To represent the position related patterns of increased muscle tone, SASRT was derived from the reactive torque in typical examples of the three groups, as shown in fig 6 . Varied shapes of reactive torque with respect to the stretch position for typical cases in the hemiparetic, parkinsonian, and normal groups are shown in panels $\mathrm{A}-\mathrm{C}$, respectively. In the representative spastic (hemiparetic) case (fig 6A), reactive torque gradually increased with respect to joint position, showing a stairwise incremental torque pattern (hereafter referred to as "step up"). In contrast, the rigidity case (fig 6B) showed an increased (relative to the norm) but constant torque pattern over the stretch range. As expected, the normal case (fig 6C) showed a consistently low reactive torque over the whole stretch range. Despite of the fact that we can easily distinguish normal muscle tone from pathological hypertonia by using ASRT, this variable is limited in its ability to differentiate spasticity from rigidity because the ASRT index represents the average reactive torque over the constant velocity range ( $\mathrm{Pl}$ to $\mathrm{P} 2$ in fig 6). The position related pattern for reactive torque- that is, the step up pattern in fig 6A (the hemiparetic case) and the persistent muscle tone in fig $6 \mathrm{~B}$ (the rigidity case)-has been averaged out. By subtracting baseline torque, the net torque curve during constant stretch (about $20-70^{\circ}$ in this case) represents the position related features during stretch (fig 6, panels D-F). In summary, net torque is presented in SASRT format with five segments that represent average reactive torque at different positions during constant velocity stretch, as shown in fig 6, panels G-I.

To analyse the position related characteristics of increased muscle tone among the hemiparetic, parkinsonian, and normal groups, the averaged patterns for all stretch velocities are shown in fig 7. Three different patterns were observed in this SASRT representation, especially at faster stretch velocities. Table 4 summarises the statistical analyses of group differences and position effects for the four different stretch velocities. The results show that group differences are significant $(\mathrm{p}<0.01)$ for all stretch velocities; a posteriori testing further revealed that three different patterns of reactive torque were distinguished using SASRT $(\mathrm{p}<0.01)$. However, stretch at $40^{\circ} / \mathrm{s}$ failed to differentiate spasticity from rigidity for position related characteristics (marked + in table 4 ). For position effects, all tests showed significant position differences for the three higher stretch velocities $(\mathrm{p}<0.01)$ but not for the $40^{\circ} / \mathrm{s}$
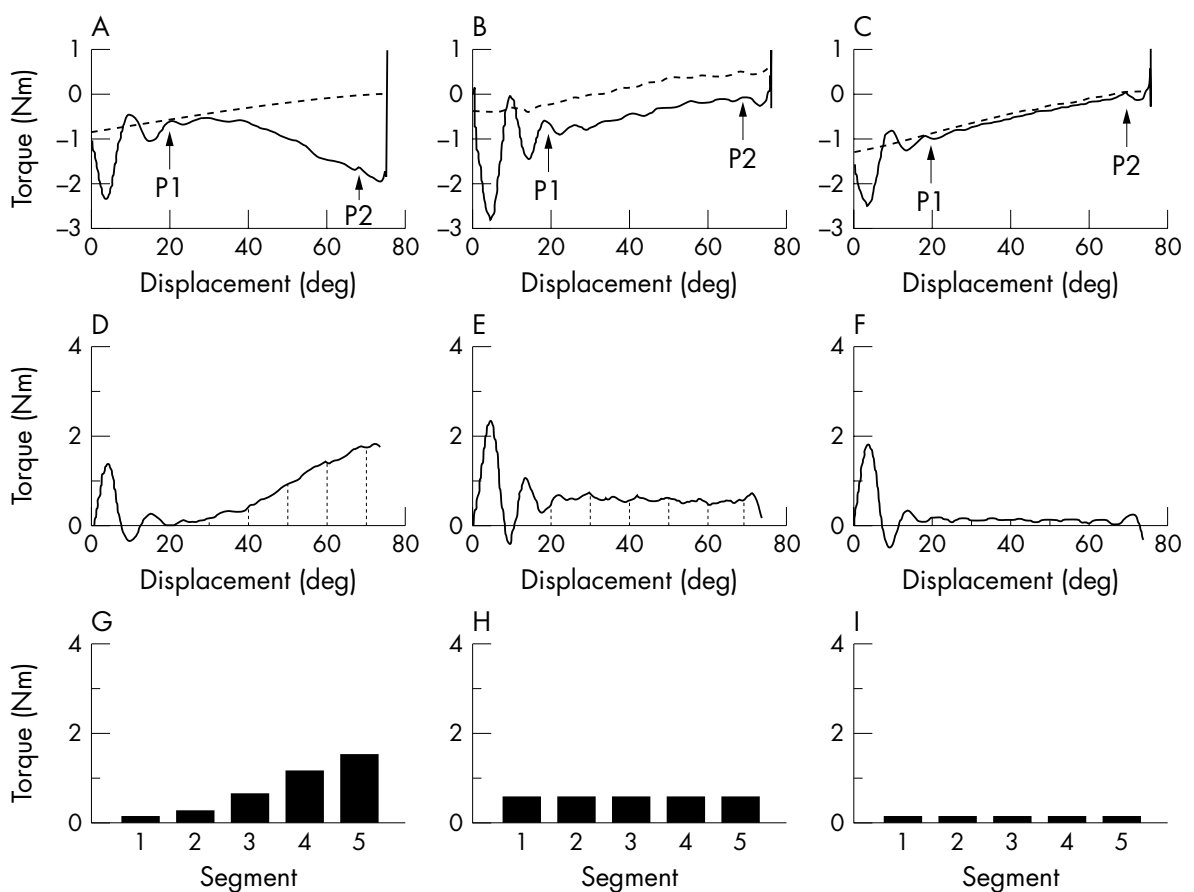

Figure 6 Formation of the position related pattern - the segmented average speed dependent reflex torque (SASRT)-was obtained from reactive torque during constant velocity stretch of $80 \%$ s (panels $A, B$, and C) by subtracting the baseline torque (panels $D, E$, and F) and further dividing into five segments (panels $\mathrm{G}, \mathrm{H}$, and I) for hemiparetic, parkinsonian, and normal subjects, respectively. Obvious differences in position dependence indicated by the SASRT index are noted in three representative cases (panels $G, H$, and I). 

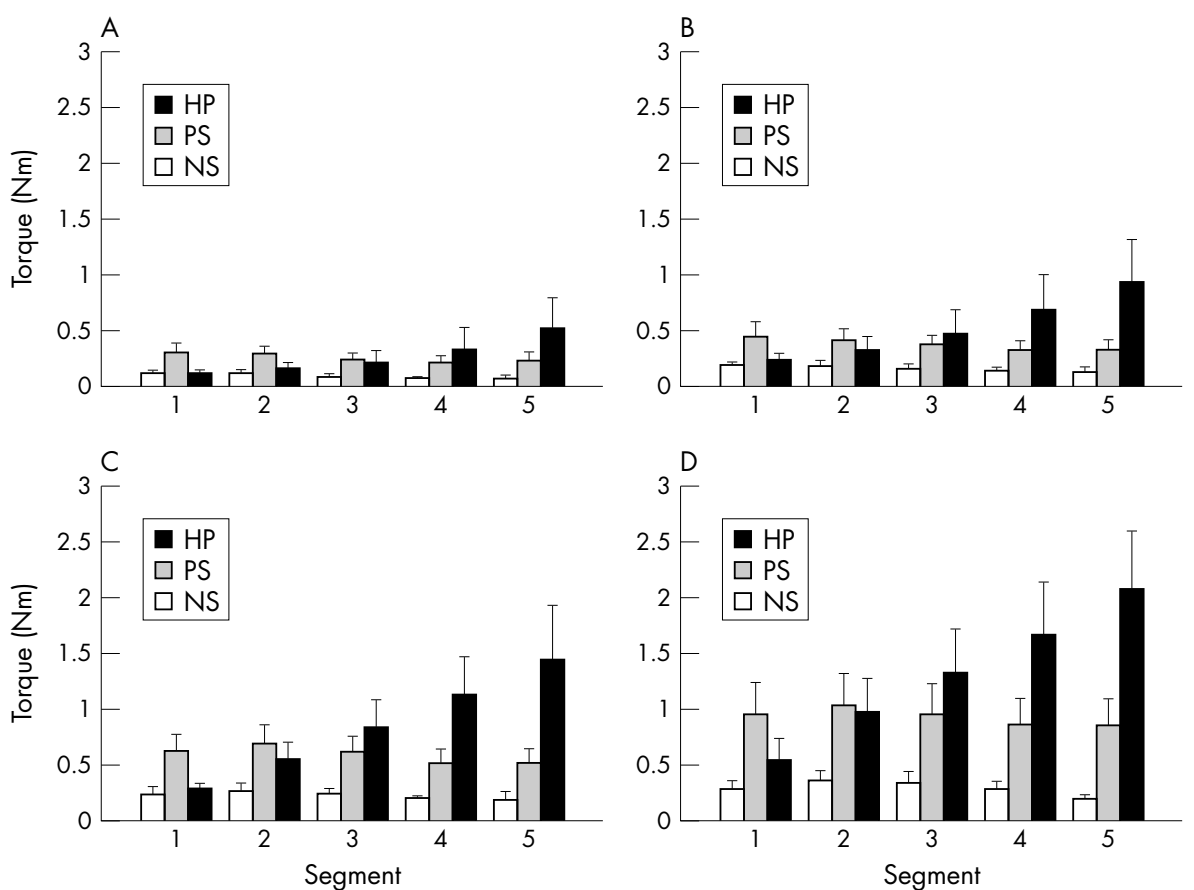

Figure 7 Position related comparison of segmented average speed dependent reflex torque (SASRT) among hemiparetic (HP), parkinsonian (PS), and normal subjects (NS) for four stretch velocities: (A) $40 \%$ s, (B) $80 \%$, (C) $120 \%$, and (D) $160 \%$. The error bar indicates the $95 \%$ confidence interval of the mean in each position segment.

stretch velocity $(\mathrm{p}>0.05$, marked with an asterisk). Significant position effects were found only for hemiparetic spasticity ( $p<0.01$, marked with a minus sign). These effects were of the step up pattern. This result indicates that the position dependent characteristic of spasticity was significant and was the main difference between the SASRT values in spasticity and rigidity.

\section{DISCUSSION}

In this study, we have presented a method for contrasting the velocity related properties of muscle tone in the elbow flexors in hemiparetic, parkinsonian, and normal subjects. ASRT, which measures the relative torque deviation from the baseline torque in a vertical stretch mode, was used for quantifying the viscous component of muscle tone at various stretch velocities. In this technique, both reflex response and passive mechanical properties contribute to the measured viscous component of the muscle tone. We excluded subjects with elbow contracture, hence it is unlikely that changes in passive properties could contribute significantly to measurements of the viscous component. The contribution of passive mechanical change, if any, should be minor and uniform during stretch, similar to that observed in the viscous component in normal subjects ( fig 6). Derived from the ASRT values of the various stretch velocities, VASRT estimates the velocity sensitivity of the measured muscle tone from repeated stretch tests. VASRT essentially measures the average viscosity, which is a viable way of quantifying the velocity dependence of increased muscle tone by considering the intrasubject variability that results from fluctuating hypertonia, ${ }^{16}$ and the intersubject variation in limb size and muscle architecture. ${ }^{18}$

To describe the position dependence of reactive torque, SASRT is used to characterise the resistance pattern during stretch. Few studies have addressed the resistance pattern during stretch because observations of position related properties are only valid in a constant velocity mode where there will be no inertial effects disturbing the torque measurement. Compared with quantification of spasticity and

Table 4 Summary of SASRT pattern comparison for stretch velocities of 40,80 , 120 , and $160^{\circ} / \mathrm{s}$

\begin{tabular}{|c|c|c|c|c|c|}
\hline & & $40^{\circ} / \mathrm{s}$ & $80 \% / \mathrm{s}$ & $120 \% \mathrm{~s}$ & $160 \% / \mathrm{s}$ \\
\hline $\begin{array}{l}\text { Group difference: } \\
\text { A posteriori test } \\
\text { (between groups): }\end{array}$ & $\begin{array}{l}H P \vee P S \\
P S \vee N S \\
H P \vee N S\end{array}$ & $\begin{array}{l}p<0.01 \\
+p>0.05 \\
p<0.01 \\
p<0.01\end{array}$ & $\begin{array}{l}p<0.01 \\
p<0.01 \\
p<0.01 \\
p<0.01\end{array}$ & $\begin{array}{l}p<0.01 \\
p<0.01 \\
p<0.01 \\
p<0.01\end{array}$ & $\begin{array}{l}p<0.01 \\
p<0.01 \\
p<0.01 \\
p<0.01\end{array}$ \\
\hline $\begin{array}{l}\text { Position effect: } \\
\text { Simple main effects } \\
\text { (position difference in) }\end{array}$ & $\begin{array}{l}\text { HP } \\
\text { PS } \\
\text { NS }\end{array}$ & $\begin{array}{l}{ }^{*} p>0.05 \\
x \\
x \\
x\end{array}$ & $\begin{array}{l}p<0.01 \\
-p<0.01 \\
p>0.05 \\
p>0.05\end{array}$ & $\begin{array}{l}p<0.01 \\
-p<0.01 \\
p>0.05 \\
p>0.05\end{array}$ & $\begin{array}{l}p<0.01 \\
-p<0.01 \\
p>0.05 \\
p>0.05\end{array}$ \\
\hline
\end{tabular}

In group difference analysis, only the hemiparesis/parkinsonism pairs in $40 \%$ s show non-significant differences (marked " + "). In position effect analysis, the position differences are only significant for the hemiparetic group (marked "-). As position effect at $40^{\circ} /$ s was not significant (marked "*"), subsequent tests (simple main effects) were not necessary (marked " $X$ ").

HP, hemiparesis; NS, normal subject; PS, parkinsonism; SASRT, segmented averaged speed dependent reflex torque. 4 
rigidity, which focus on the amplitude of increased muscle tone using ASRT as an index, observations of position related resistance provide some interesting information for comparing these two types of increased muscle tone.

In investigating spasticity, the underlying pathophysiology - that is, the hyperexcitability of the stretch reflex in response to passive stretch-can be observed from ASRT, VASRT, and SASRT indices. Compared with normal control, a higher ASRT (table 2) was observed in hemiparetic limbs, which implies an enhanced stretch response in spasticity. This pathological enhancement of muscle tone generally corresponds to the common concept of increased stretch reflex gain in the spastic elbow. ${ }^{54}$ From our study, we can observe this enhanced stretch reflex even at a low stretch velocity (that is, $40 \%$ ). This observation reflects the fact that the stretch reflex of the spastic muscle is more sensitive to stretch velocity ${ }^{5}$ than normal muscle.

From the VASRT analyses, our study showed that VASRT in the spastic limb was more prominent than in the normal subjects. A higher VASRT in the spastic subjects indicated a substantial increase in reactive torque in response to the increase in stretch velocity. These observations correspond to the velocity dependent feature of the stretch reflex found in other studies. ${ }^{5-8}$ Furthermore, SASRT analysis showed that muscle resistance starts at a relatively small level and, after a certain point, increases progressively with increasing muscle torque during the stretch (as seen in figs 6 and 7). Similar stretch response waveforms were previously observed in spastic elbow joint stretch and were interpreted as reflecting the onset of, and the subsequent increase in, stretch reflex activities. ${ }^{65-28}$ During larger stretch amplitude $\left(75^{\circ}\right.$ in our study), the stretch reflex activity possibly continues and increases in a position dependent fashion until the stretch stops. ${ }^{5}$ The observed resistance pattern, with latency delayed and increased proportional to stretch amplitude, generally correlates with the clinical perception of stretching a spastic limb. ${ }^{29}$

Similar quantitative measurements were applied to observations of the velocity dependent and position dependent properties of parkinsonian rigidity. From ASRT and VASRT analyses, our results suggest that enhanced muscle tone and velocity dependence of muscle resistance are also observed in parkinsonian rigidity (tables 2 and 3). In empirical observation, spasticity is hypothesised to be velocity dependent, but parkinsonian rigidity is velocity independent. However, our analysis of VASRT showed there was no significant difference between spasticity and rigidity (table 3 ). Although no research has directly studied the velocity dependence of parkinsonian rigidity, several studies have shown circumstantial evidence for velocity effects on stretched rigid muscles in parkinsonian subjects. Higher stretch responses in knee extensors ${ }^{30}$ and ankle plantar flexors ${ }^{31}$ were found for higher stretch velocities. Similar research has reported that a larger torque hysteresis loop area during passive ankle stretch can be found at higher stretch velocities in patients with Parkinson's disease than in normal subjects. ${ }^{8}$ Other studies have indicated that stretch velocity is an important factor differentiating rigidity from normal muscle tone. ${ }^{32}{ }^{33}$ Although the exact pathways involved in the genesis of rigidity are still not clear, involvement of the reflex mechanism is generally assumed by investigators. ${ }^{10} 12$ Our ASRT and VASRT findings suggest that enhanced gain and pathological velocity dependence of the reflex response are also notable in parkinsonian rigidity.

Although ASRT and VASRT were increased in both hemiparetic and parkinsonian subjects, parkinsonian rigidity further features a position independent property observed from SASRT, as shown in figs 6 and 7. Even at the highest stretch velocity used in this study $\left(160^{\circ} / \mathrm{s}\right)$, the increase in muscle resistance in parkinsonian rigidity did not correlate with the various stretch positions. This consistent resistance conforms to general conceptions and previous clinical experience, $^{12}{ }^{34}$ and is presumed to be a result of overactive tonic reflex contraction. ${ }^{35}$ Our SASRT analysis also showed that position dependent properties during stretch can be used to differentiate rigidity from spasticity (table 4 ), especially at higher stretch velocities $\left(80,120\right.$, and $\left.160^{\circ} / \mathrm{s}\right)$. Although sharing similar features of supraspinal origins and reflex mechanisms, the pathological neural control of muscle length change is quite different in spastic and rigid limbs according to our SASRT findings. Before inducing a reflex contraction, rigidity-unlike spasticity-begins with increased muscle tone without latency. Our observation of sustained and position independent resistance for rigidity implies that rigid muscle tone is independent of the increased segmental stretch reflex that regulates spastic change in muscle length. Parkinsonian rigidity is more complex than the segmental reflex loop, and its pathophysiological mechanism is still not clearly understood, although additional long loop reflex pathways $^{10}{ }^{34}{ }^{36-37}$ and pre-existing $\alpha$ motor neurone overactivity from defective supraspinal $\operatorname{control}^{36-38}$ have been suggested.

\section{Conclusions}

In this study we have provided data which support quantitatively our general conceptions and clinical observations of spasticity, rigidity, and normal muscle tone. Our results suggest that velocity dependent properties are significant in both spastic and rigid elbow flexors when we stretch them passively. However, the progressively increasing muscle tone of spasticity is different from the increased (relative to normal) but constant muscle tone found in parkinsonism. The results of the study may be of interest to clinicians and researchers dealing with spasticity and rigidity. In addition, further studies can extend the findings to other muscle groups, where the effects of muscle stretch might differ from those of the elbow flexors.

\section{ACKNOWLEDGEMENTS}

This research was financially supported by grants from National Science Council and National Health Research Institute, ROC, under grants NSC 89-2213-E-006-041 and NHRI-EX90-9017EP.

\section{Authors' affiliations}

H-M Lee, Institute of Biomedical Engineering, National Cheng Kung University, Tainan, Taiwan, ROC

Y-Z Huang, Department of Neurology, National Cheng Kung University and Sobell Department of Motor Neuroscience \& Movement Disorders, Institute of Neurology, Queen Square, London, UK

J-J J Chen, Institute of Biomedical Engineering, National Cheng Kung University

I-S Hwang, Department of Physical Therapy, National Cheng Kung University

\section{REFERENCES}

1 Kamper DG, Rymer WZ. Quantitative features of the stretch response of extrinsic finger muscles in hemiparetic stroke. Muscle Nerve 2000;23:954-61.

2 Rea GL, Ebner TJ. Quantitative assessment of the effect of basal ganglia lesions on the stretch reflex in primates. Stereotact Funct Neurosurg 1991;56:44-65.

3 He J, Norling WR, Wang Y. A dynamic neuromuscular model for describing the pendulum test of spasticity. IEEE Trans Biomed Eng 1997;44:175-84

4 Lance JW. Symposium synopsis. In: Feldman RG, Young RR, Koella WP eds. Spasticity: disordered motor control. Chicago: Year Book Medical Publishers, 1980:485-94.

5 Thilmann AF, Fellows SJ, Garms E. The mechanism of spastic muscle hypertonus. Variation in reflex gain over the time course of spasticity. Brain 1991;114:233-44.

6 Powers RK, Campbell DL, Rymer WZ. Stretch reflex dynamics in spastic elbow flexor muscles. Ann Neurol 1989;25:32-42.

7 Firoozbakhsh KK, Kunkel CF, Scremin AM, et al. Isokinetic dynamometric technique for spasticity assessment. Am J Phys Med Rehabil 1993;72:379-85.

8 Broberg C, Grimby G. Measurement of torque during passive and active ankle movements in patients with muscle hypertonia. A methodological study. Scand J Rehabil Med 1983;9:108-17.

9 Dietrichson P. Phasic ankle reflex in spasticity and Parkinsonian rigidity The role of the fusimotor system. Acta Neurol Scand 1971;47:22-51.

10 Lee RG. Pathophysiology of rigidity and akinesia in Parkinson's disease. Eur Neurol 1989;29:13-18. 
11 Bergui M, Lopiano L, Paglia G, et al. Stretch reflex of quadriceps femoris and its relation to rigidity in Parkinson's disease. Acta Neurol Scand 1992;86:226-9.

12 Dimitrijevic MR. Spasticity and rigidity. In: Jankovic J, Tolosa E, eds. Parkinson's disease and movement disorders, 3rd ed. Baltimore: Williams and Wilkins, 1998:159-75.

13 Wiegner AW, Watts RL. Elastic properties of muscles measured at the elbow in man. I. Normal controls. J Neurol Neurosurg Psychiatry 1986:49:1171-6.

14 Watts RL, Wiegner AW, Young R. Elastic properties of muscles measured at the elbow in man. II. Patients with Parkinsonian rigidity. $J$ Neurol Neurosurg Psychiatry 1986;49:1177-81.

15 Caligiuri MP, Galasko DR. Quantifying drug-induced changes in parkinsonian rigidity using an instrumental measure of activated stiffness. Clin Neuropharmacol 1992;15:1-12.

16 Prochazka A, Bennett DJ, Stephens M, et al. Measurement of rigidity in Parkinson's disease. Mov Disord 1997; 12:24-32.

17 Toft E. Mechanical and electromyographic stretch responses in spastic and healthy subjects. Acta Neurol Scand 1995;163:1-24.

18 Ju MS, Chen JJ, Lee HM, et al. Time-course analysis of stretch reflexes in hemiparetic subjects using an on-line spasticity measurement system. J Electromyogr Kinesiol 2000;10:1-14.

19 Brown RA, Lawson DA, Leslie GC, et al. Does the Wartenberg pendulum test differentiate quantitatively between spasticity and rigidity? A study in elderly stroke and Parkinsonian patients. J Neurol Neurosurg Psychiatry 1988;51:1178-86.

20 Thilmann AF, Fellows SJ, Garms E. Pathological stretch reflexes on the "good" side of hemiparetic patients. J Neurol Neurosurg Psychiatry 1990;53:208-14.

21 Kearney RE, Hunter IW. System identification of human joint dynamics. Crit Rev Biomed Eng 1990;18:55-87.

22 Hershler C, Milner M. An optimality criterion for processing electromyographic signals relating to human locomotion. IEEE Trans Biomed Eng 1978;25:413-20.

23 Gabel RH, Brand RA. The effects of signal conditioning on the statistical analyses of gait EMG. Electroencephalogr Clin Neurophysiol 1994;93:188-201

24 Katz RT, Rymer WZ. Spastic hypertonia: mechanisms and measurement. Arch Phys Med Rehabil 1989;70:144-55.
25 Powers RK, Marder-Meyer J, Rymer WZ. Quantitative relations between hypertonia and stretch reflex threshold in spastic hemiparesis. Ann Neurol 1988;23:115-24.

26 Katz RT, Rovai GP, Brait C, et al. Objective quantification of spastic hypertonia: correlation with clinical findings. Arch Phys Med Rehabil 1992;73:339-47.

27 Schmit BD, Dhaher Y, Dewald JPA, et al. Reflex torque response to movement of the spastic elbow: theoretical analyses and implications for quantification of spasticity. Ann Biomed Eng 1999;27:815-29.

28 Pisano F, Miscio G, Del Conte C, et al. Quantitative measures of spasticity in post-stroke patients. Clin Neurophysiol 2000;111:101522.

29 Andrews CJ, Burke D, Lance JW. The response to muscle stretch and shortening in Parkinsonian rigidity. Brain 1972;95:795-812.

30 Gregoric M, Stefanovska A, Vodovnik L, et al. Rigidity in parkinsonism: characteristics and influences of passive exercise and electrical nerve stimulation. Funct Neurol 1988;3:55-68.

31 Gresty M. Stability of the head in pitch (neck flexion-extension): studies in normal subjects and patients with axial rigidity. Mov Disord 1989;4:233-48.

32 Teräväinen $\mathbf{H}$, Tsui JK, Mak E, et al. Optimal indices for testing parkinsonian rigidity. Can J Neurol Sci 1989;16:180-3.

33 Rothwell JC, Obeso JA, Traub MM, et al. The behaviour of the long-latency stretch reflex in patients with Parkinson's disease. J Neurol Neurosurg Psychiatry 1983:46:35-44.

34 Marsden CD. Motor dysfunction and movement disorders. In: Asbury AK, Mckhann GM, McDonald WI, eds. Disease of the nervous system. Clinical neurobiology. Philadelphia: WB Saunders, 1992:309-18.

35 Berardelli A, Sabra AF, Hallett M. Physiological mechanisms of rigidity in Parkinson's disease. J Neurol Neurosurg Psychiatry 1983:46:45-53.

36 Lee RG, Tatton WG. Motor responses to sudden limb displacements in primates with specific CNS lesions and in human patients with motor system disorders. Can J Neurol Sci 1975;2:285-93.

37 Marsden CD. The mysterious motor function of the basal ganglia: the Robert Wartenberg Lecture. Neurology 1982;32:514-39.

38 Cantello R, Gianelli M, Civardi C, et al. Parkinson's disease rigidity: EMG in a small hand muscle at "rest". Electroencephalogr Clin Neurophysiol 1995;97:215-22. 